\title{
Quantitative Valuation Analysis of Surface Area of Low-dimensional Nanomaterials Based on Sensors
}

\author{
Qiang Zou ${ }^{1, \mathrm{a}}$, Shentong Mo ${ }^{1, \mathrm{~b}}$, Guohui $\mathrm{Li}^{2, \mathrm{c}, ~ *}$ and Bing $\mathrm{Yu}^{3,4, \mathrm{~d}}$ \\ ${ }^{1}$ School of Mircoelectronics, Tianjin University, Weijin Road, Tianjin, China \\ ${ }^{2}$ Tianjin Development Zone Aojin High New Technology co., LTD, Weijin Road, Tianjin, China \\ ${ }^{3}$ School of Environmental Science and Engineering, \\ Tianjin University, Weijin Road, Tianjin, China \\ ${ }^{4}$ Shanghai Yanhua Smartech Group, Shanghai, China \\ a moshentong678@126.com, ${ }^{\mathrm{b}}$ zouqiang@tju.edu.cn, ${ }^{\mathrm{c}}$ liguohui@tju.edu.cn, ${ }^{\mathrm{d}}$ yubing@tju.edu.cn \\ *corresponding author
}

Keywords: Zero-dimension, One-dimension, Nanomaterials, Surface area, Nanoparticles

Abstract. In this paper, a general method of producing zero-dimensional (0-D) and onedimensional (1-D) nano-structures by adjusting only one parameter in the process of production is investigated. To further study the result of small-scale effects, a quantitative characterization for the specific surface area of 1-D nanostructure is proposed. And the characterization method could be integrated into the nano-systems to reflect useful parameters for the analysis of nanomaterials. These two methods mentioned above could enhance nano-fabrication in volume productions, as well as predict the properties of nanodevices in years to come. This means, to some extent, would exert an unquestionably positive impact on manufacturing of nanomaterials and nanodevices.

\section{Introduction}

Nanotechnology has been highly applied to different fields and playing crucial role in scientific development [1-4]. The key parameters of nanodevices are determined by surface area of certain nanostructures [5-6]. The specific surface area of nano-scaled substrate can be raised by several orders of magnitude [7-9]. 1D-nanostructure can expand surface area of every substrate. And the production of 1D-nanostructure with controllable method of processing [10-12] is attracting more and more attention. The application of nanostructures could be largely enhanced once nanostructures are precisely characterized with a new method of fractal theory (as shown in Figure 1) $[13-15]$. 


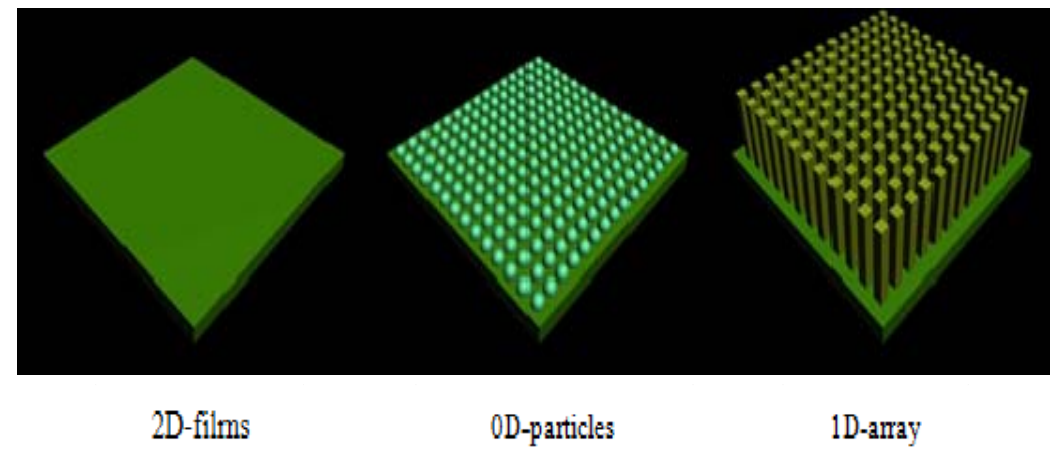

Figure 1 The surface of different nano-sized dimensional structure characteristics on the same substrate

Nowadays, most of nanodevices need to be made into a finished product to measure the specific surface area of them [16-18]. In this paper, we propose a method of measurement that the nanodevices can be measured by semi-finished products [19]. Compared to the existing method, the proposed method greatly reduces the cost and risk to optimize the producing method [20].

\section{Experimental}

Polished single-crystal Si chip is chosen as the substrate material. In the magnetron sputtering process, the $\mathrm{Zn}$ target is of $99.999 \%$ high purity, and the Si surface is coated with a $\mathrm{Zn}$ film in thickness of $500 \mathrm{~nm}$. Heating at $400^{\circ} \mathrm{C}$ for $2 \mathrm{~h}$, the Si substrate is coated with a layer of epitaxial $\mathrm{ZnO}$ on the surface. Polyvinyl alcohol (PVA) and deionized water are mixed up at the mass ratio of 4 : 100, putting aside for $48 \mathrm{~h}$ at $30^{\circ} \mathrm{C}$. Transparent PVA mixture solution is obtained; the $\mathrm{Zn}(\mathrm{AC})_{2}$ and PVA solution is mixed at the mass ration of $\mathrm{Zn}(\mathrm{AC})_{2}$ : PVA = 1:3 (sample a), 1:2 (sample b), 1:1 (sample c), 2:1 (sample d), 3:1 (sample e), 4:1 (sample f). At the same time, the mixing process needs water bath heating at $90^{\circ} \mathrm{C}$ and mechanical stirring for $1-2 \mathrm{~h}$. The Si substrate with an epitaxial $\mathrm{ZnO}$ layer is immersed into solution, tiling at the bottom of mixed liquid. The mixture is placed in the $120^{\circ}$ Coven for $4-5 \mathrm{~h}$ (this course existed or no, it is the different of samples in 0Dnanostructure or 1D-nanostructure), and then transferred into to muffle furnace, heated at $600^{\circ} \mathrm{C}$ for 3-5 $\mathrm{h}$. After that, the sample could be moved out and cooled to room temperature in air.

The gray value of Scanning Electron Microscope (SEM) image of nanomaterials is different from the real height of the samples in 0D and 1D nanostructures. Based on the assumption of Pentland, the difference has been considered. Even the changes of resolution are limited in some scope, the objects in nature and their corresponding image still have a certain degree of correlation. So according to their gray change of SEM images, it is possible to estimate the surface area of nanomaterials.

Early researches have proved that low-dimensional nanomaterials have obvious fractal characteristics. According to the fractal theory, the measurement results will become more and more precise. With the reduction of measurement, they tend to physical limits. And measurement results and scales approximately meet linear relationship in dual logarithmic axis. Thus, the measurement result for the surface area of nanomaterials is different from that of the real project. In this paper, the method of data fitting is used to solve this problem according to fractal theory. Assuming that nanomaterials meet Fractional Brownian Motion (FBM) model, when measure step reaches minimum, there is a limit (LB) of surface area. The LB can be reached with a series of measurement results in different scales. We strongly encourage authors to use this document for the preparation of the camera-ready. Please follow the instructions closely in order to make the volume look as uniform as possible. 


\section{Results and discussion}

A group of measurement results of surface area in different scales were taken as raw data. The measurement results were fitted in logarithmic axis with the method of Least Squares in this paper. When the measurement scale is close to minimum, the interception of fitting line in vertical axis is the logarithmic limit of surface area. According to the gray change of their SEM images, the surface area of materials in different scales can be estimated. The measurement results in different scales are shown in Figure 2.

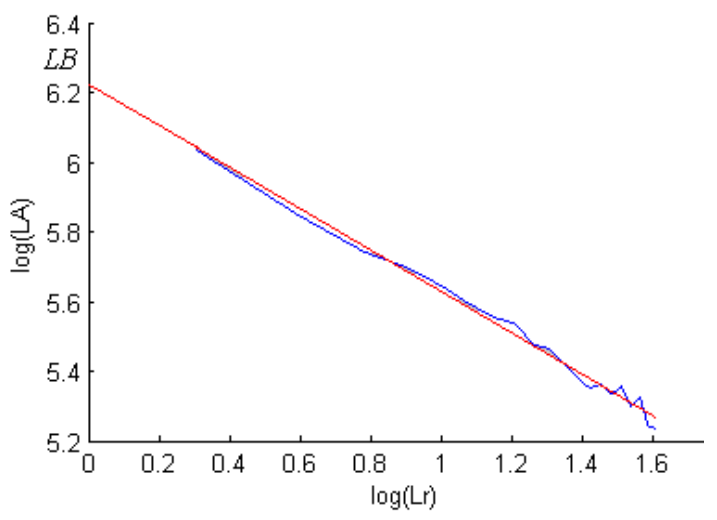

Figure 2 Measurement results of some low-dimensional nanomaterials and their double logarithmic linear fitting results in different scales

Taking Figure 2 as example, $\mathrm{LB}=6.2240$, the specific surface area parameter of nanomaterials can be calculated according to LB. And it is defined as Eq. (1).

$$
K=\exp (L B) / S \text {. }
$$

Where LB is the logarithmic limit of surface area, S is the total area of SEM image. The physical meaning of the parameter $\mathrm{K}$ for specific surface area can be regarded as surface area of lowdimensional nanomaterials with unit basal area.
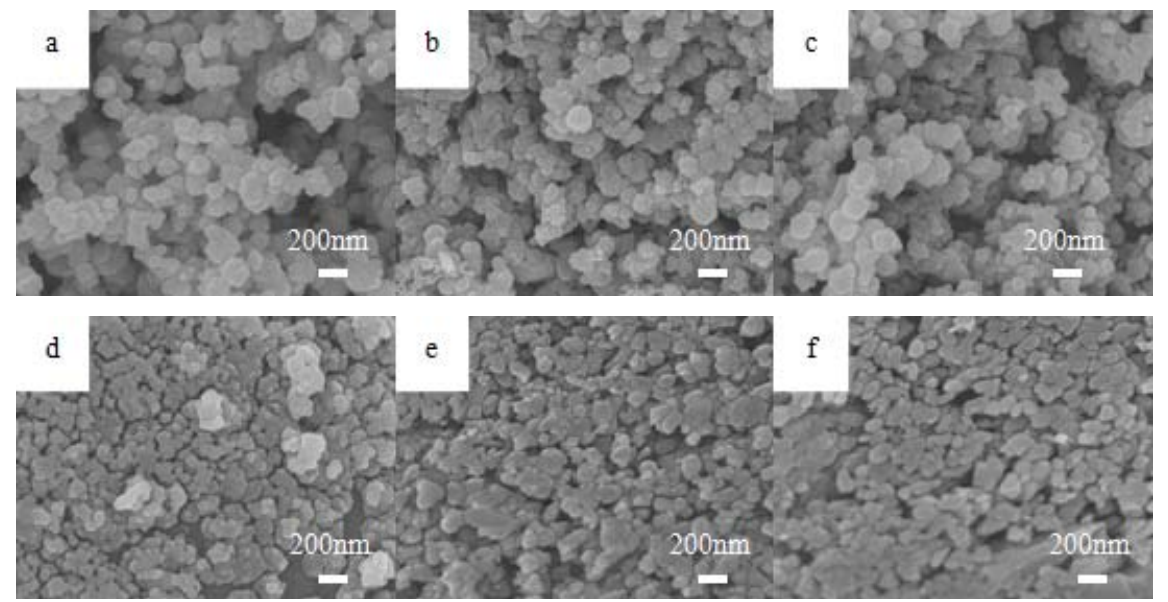

Figure 3 SEM images of several kinds of 0D-nanomaterial

In this paper, 6 SEM images of 0D-nanostructure with the same basal area (magnification is 40,000) were selected as shown in Figure 3. And 6 SEM images of 1D-nanostructure with the same basal area (magnification time is 10,000) were selected in this paper. They are shown in Figure 4. 


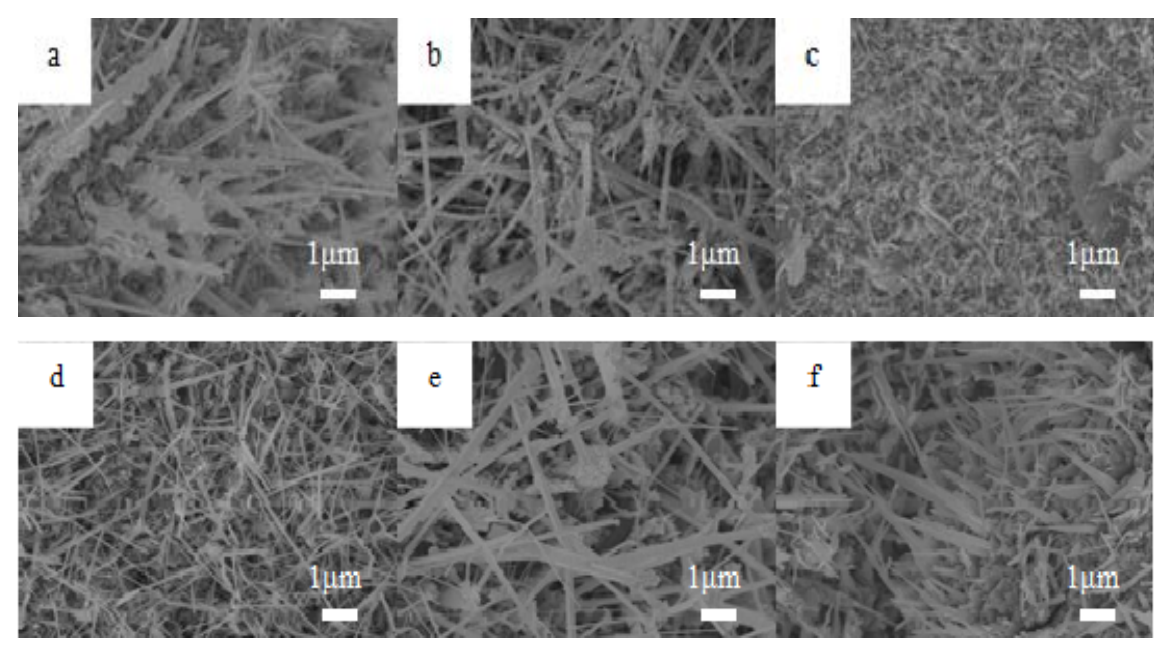

Figure 4 SEM images of several kinds of 1D-nanomaterial

To analyse the surface area of traditional nanodevices, the specific surface area of nanoparticles can be detected by the traditional method, such as laser particle size analyzer. But, up to now, the special structures with the surface of nanodevices covered by complex nanoparticles and 1Dnanomaterial, have been calibrated by no mature technology. Since there is no mature mapping technology, the surface area of nanodevices could not be measured with standard equipment, as shown in Figure 3 and Figure 4. On the other hand, it is impossible to predict the performance of the devices based on the size of specific surface area of nanodevices. Therefore, it is urgent to analyse the image by the equipment based on detection image, such as the SEM. And it will provide a suitable method for the prediction of the calibration of nano projection and the performance of nanosensors. The traditional particle size analyzer is only an analysis of the specific surface area of discrete nanoparticles. And the structure, such as the surface of the sensor covered by nanoparticles could not be detected and calibrated. At the same time, the surface of sensors covered by the nanostructures such as 1D-nanoparticle and 1D-nanowire, could not be calibrated by mature technology.

From Figure 5, it can be seen clearly that the specific surface area parameters of 1D-nanomaterial are much higher than them of 0D-nanomaterial. For 6 different structures of 0D-nanomaterial, their calculation results are in accordance to the estimation results of human eyes. Besides that, for 6 different structures of 1D-nanomaterial, a new estimation method of structure of materials was proposed. Based on fractal theory, this method was wonderfully verified in this paper. 


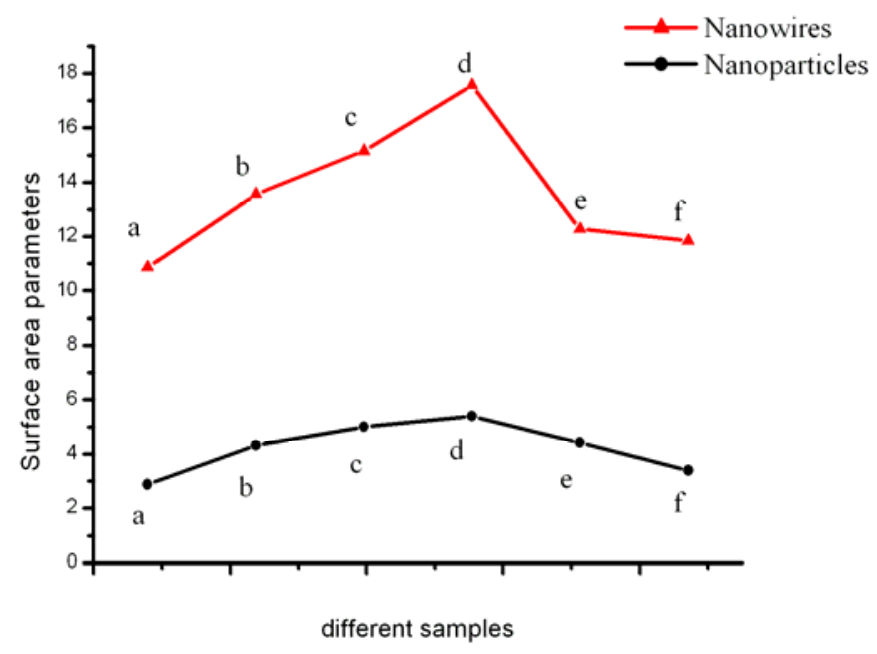

Figure 5 Results of specific surface area of $0 \mathrm{D}$ and 1D nanomaterials

\section{Conclusions}

In this paper, based on the fractal theory, the SEM images of nanomaterials in different shapes were calculated and analysed. And then a method to value surface of samples was proposed to estimate the specific surface area of low-dimensional nanomaterials quantitatively. By adjusting only one parameter in the process of producing, the 0D-nanostructure and 1D-nanostructure could be produced with good control. Nanostructures could be better characterized and the properties of nanodevices could be predicted in the future. The request for such a cantilever structure will be in great demands. The great demands not only save us huge funds, but also provide the society with a big impulse by expanding the application of nanostructures in production and measurements.

\section{Acknowledgements}

This work was supported by Ministry of Science and Technology of the People's Republic of China (Contract No. 2015BAJ01B01), and Special Project of Tianjin University in Wuqing District (2013), as well as sponsored by Center for post-doctoral studies in Tianjin Development Zone Aojin High New Technology co., LTD.

\section{References}

[1] Abu Sadat Mohammad Iftekhar Uddin, Duy-Thach Phan and Gwiy-Sang Chung, (2014) Synthesis of Zno Nanoparticles-reduced Graphene Oxide Composites and Their Intrinsic Gas Sensing Properties [J]. Surf. Rev. Lett, 21(6), 1450086-1450097.

[2] C. S. LIM, M. H. HONG, Y. LIN, L. S. TAN and A. SENTHIL KUMAR. (2010) Large Area Parallel Surface Nanostructuring with Laser Irradiation through Microlens Arrays [J]. Surf. Rev. Lett, 17(03), 383-387.

[3] Shaohua Zheng, Ruifang Guan, Qiuju Guo and Dengcheng Su. (2007) The Design of Surface Modification of Amorphous Zro2 Nanoparticles as A Lubricant Additive [J]. Surf. Rev. Lett, 14(14), 1047-1052.

[4] R. M. Feenstra, Huajie Chen, V. Ramachandran C. D. LEE, A. R. SMITH, J. E. Northrup, T. Zywietz, J. Neugebauer and D. W. Greve. (2012) Surface Morphology of Gan Surfaces During Molecular Beam Epitaxy [J]. Surf. Rev. Lett, 07(5n06), 601-606. 
[5] Sanjun Wang, Youlin Song, Xianlin Zhao, Xianjun Wang, JINHAI LIU, JINMING LI and YU JIA. (2006) The Calculation of the Surface Energy of High-index Surfaces of Silicon at Zero Temperature [J]. Surf. Rev. Lett, 13(6), 841-846.

[6] Dongseok Park, Youngjo Tak, Jaehyun Kim and Kijung Yong. (2007) Low-temperature Synthesized Zno Nanoneedles: Xps and Pl Analysis [J]. Surf. Rev. Lett, 14(06), 1061-1065.

[7] C.-H. Nien and T. E. Madey. (1999) Surface Reconstructions and Morphological Modifications: Restructuring of W(111) Induced by Sulfur Overlayers [J]. Surface Science, 433(99), 254-260.

[8] Jacques Jupille and R Lazzari. (2006) Improving the Wetting of Oxides by Metals [J]. Journal of Optoelectronics \& Advanced Materials, 8(3), 901-908.

[9] Vuk Uskoković and Miha Drofenik. (2005) Synthesis of Materials within Reverse Micelles [J]. Surf. Rev. Lett, 12(2), 239-277.

[10] Enling Li, Xuewen Wang, Shanshan Wang and Guican Chen. (2012) Characteristic Analysis on Gan Nanocrystalline Powder Prepared by Sol-gel Method [J]. Surf. Rev. Lett, 14(06), 1181-1185.

[11] X. J. Zhang, X. H. Zhang, B. Wang, C Zhang, JC Chang, Chun Sing Lee and Shuit-Tong Lee. (2013) One- or Semi-Two-Dimensional Organic Nanocrystals Induced by Directional Supramolecular Interactions [J]. J. Phys. Chem. C, 112(42), 16264-16268.

[12] J. R. Wendt, GA Vawter, RE Smith and ME Warren. (1995) Nanofabrication of Subwavelength, Binary, High-efficiency Diffractive Optical Elements in GaAs [J]. J. Vac. Sci. Technol. B, 13(6), 2705-2708.

[13] X. J. Zhang, X. H. Zhang, K. Zou A. C. Lee and S. T. Lee. (2007) Single-Crystal Nanoribbons, Nanotubes, and Nanowires from Intramolecular Charge-Transfer Organic Molecules [J]. J. Am. Chem. Soc, 129(12), 35273532 .

[14] P. Kirsch, MB Assouar, O Elmazria, V Mortet and P Alnot. (2006) 5GHz Surface Acoustic Wave Devices Based on Aluminum Nitride/Diamond Layered Structure Realized Using Electron Beam Lithography [J]. Appl. Phys. Lett, 88(88), 223504-223504-3.

[15] C. J. Bhongale, C. W. Chang, C. S. Lee, WG D and CS Hsu. (2005) Relaxation Dynamics and Structural Characterization of Organic Nanoparticles with Enhanced Emission [J]. J. Phys. Chem. B, 109(28), 13472-13482.

[16] J. A. van Kan, A. A. Bettiol and F. Watt. (2003) Three-dimensional Nanolithography Using Proton Beam Writing [J]. Appl. Phys. Lett, 83(83), 1629-1631.

[17] E. Kwon, H. R. Chung, Y. Araki, H Kasai, H Oikawa, Osamu Ito and Hiroshi Nakanishi. (2007) Surface Structure Effect on Optical Properties of Organic Nanocrystals [J]. Chem. Phys. Lett, 441(1-3), 106-108.

[18] C. S. Lim, MH Hong, Y Lin, Q Xie and BS Lukyanchuk. (2006) Microlens Array Fabrication by Laser Interference Lithography for Super-resolution Surface Nanopatterning [J]. Appl. Phys. Lett, 89(19), 191125191125-3.

[19] J. S. Hu, Y. G. Guo, H. P. Liang, LJ Wan and L Jiang. (2005) Three-dimensional Self-organization of Supramolecular Self-assembled Porphyrin Hollow Hexagonal Nanoprisms [J]. J. Am. Chem. Soc., 127(48):17090-17095.

[20] J. Mori, Y. Miyashita, D. Oliveira H Kasai, H Oikawa and Hachiro Nakanishi. (2009) Stopped-flow Analysis on the Mechanism of Perylene Nanoparticle Formation by the Reprecipitation Method [J]. J. Cryst. Growth, 311(3), 553-555. 